\title{
Papyrological Investigations: Transferring Perception and Interpretation into the Digital World
}

\author{
Ségolène M. Tarte*
}

\begin{abstract}
Deciphering ancient and damaged documents is a complex investigative task that papyrologists routinely undertake to extract meaning from the script. Perception and interpretation play an essential role. In this paper, we present methods for transferring to the digital world some of the processes that experts draw upon when interpreting a text, with the ultimate aim of constructing an Interpretation Support System (ISS) for papyrologists. Image-capture and image-processing approaches that reflect real-world perceptual processes have been implemented. In addition, we propose an expansion of a previously built model of papyrological reading and transcription. We make explicit some of the implicit processes involved in an interpretation effort, using an example where papyrologists developed hypotheses for the identification of a puzzling letter form. Two distinct yet not mutually exclusive approaches to the interpretation task have been identified: the kinaesthetic/palaeographical strategy and the cruciverbalistic/philological strategy. The ISS will have to facilitate both approaches. Mechanisms triggering the emergence of working hypotheses of interpretation, which we call percepts, have also been pinpointed; they include skilled vision, scholarly expectations, aspect shifting and local-global oscillations. Working hypotheses being triggered by such mechanisms can then be exposed as an explicit network of sourced percepts; these mechanisms also confer a qualitative well-foundedness to the percepts and hence help us to retrace and assess the rationale leading to a specific interpretation.
\end{abstract}

Forthcoming in Literary and Linguistic Computing, Special Edition on Digital Objects, 2011

*Oxford e-Research Centre, University of Oxford, UK. segolene.tarte@oerc.ox.ac.uk 


\section{Introduction}

The daily work of papyrologists consists of transcribing ancient texts. Such ancient texts are primarily written on papyrus, but also commonly on wood, potsherds or metal. Classical historians generally work on texts in Greek or Latin, but may also acquire expertise in other languages such as Coptic. Their primary task is one of deciphering and transcribing scarcely legible texts; a task whose complexity is increased by the need to develop an interpretation of the texts in order to extract their meaning (Youtie, 1963). The difficulties of interpreting ancient texts are multiple. Damage and stains frequently obscure the writing; the text might be incomplete; the document can be a palimpsest. Hence papyrologists undertaking a task of interpretation rely heavily upon perception. Further, the interpretation of a document rarely happens within a single session; when they set to the task again, or want to share and discuss the outcome of their efforts, remembering why and how they have come to commit to a specific piece of interpretation often requires them to reconstruct the rationale that led them to their working hypotheses. Overall, a papyrologist's daily work resembles detective work. It involves detecting clues, elaborating hypotheses, setting out argumentation and justifying each commitment to a given hypothesis.

Our aim is to assist papyrologists in their investigations, by developing a web-based software tool that will enable them both to transcribe the script more easily and to document the trail of their interpretation effort. To that end, we have studied how papyrologists work and focused on transferring some of their real-world perception and interpretation mechanisms and processes into the digital world. This work was conducted within the framework of the 'Image, Text, Interpretation: Imaging, Technology and Documents' project (or, for short, eScience and Ancient Documents-eSAD ${ }^{1}$ ), which aims to build an Interpretation Support System (ISS) that will ultimately enable papyrologists to make explicit some of the implicit methods, processes and knowledge that are deployed throughout an interpretation task.

Taking as a guiding thread the narrative of how three papyrologists jointly unravelled the meaning of a specific Roman wooden stylus tablet, which is introduced in section 2, sections 3 and 4 demonstrate how some of the real-world perceptual processes have been successfully recreated and transposed to the digital world. Section 5 attempts to unpack the intricate rationale that led to the emergence of a particular piece of interpretation, thus illustrating our initial steps towards an understanding of the papyrologist's detective work. This approach aims to inform our design decisions for future porting into the digital world. It must be stressed here, that the aim of the ISS that is being developed is not to automate the interpretation process, but rather to facilitate the digital recording and tracking of the unravelling of that process.

\section{The Frisian Tablet}

The type of writing materials under scrutiny are wooden stylus tablets, as used in Classical Antiquity. Such a tablet presents itself as a thin block of wood (usually silver fir or cedar) the size of a modern postcard in which a shallow recess was carved. The recess was coated with wax and the text was inscribed in the wax with a sharp metallic stylus. The incisions 
often penetrated the coat of wax and left scratch and score marks of various depths in the wood. In most cases, when found, the wax surface of the tablet has perished, and the text must be read from the scores in the wood. Texts were usually spread over several such tablets, which were then bound together and sometimes sealed. The conditions in which those tablets have survived usually creates stains and damage, and in most cases, the text is incomplete.

The Frisian tablet reported upon in this paper allowed us to observe three papyrologists at work. It is a damaged and stained tablet, inscribed on both sides with a Latin cursive script, and it forms only part of the original document. It was found in 1914 in Frisia (in Tolsum, Netherlands), and the first edition of its transcription was made in 1917 by a respected scholar (Vollgraff, 1917). It was an isolated find, so its archaeological context is non-existent. The dating of the tablet to the first century $A D$ based on the reading of a consulship was originally very insecure, and although a Carbon14 dating test was conducted in 1998 (Slob, 1998), the window for its date remained large. It was decided in 2007 to attempt to clarify further the reading of the date of the tablet by taking advantage of recent imaging technology and ICT tools. Three papyrologists convened in six sessions to work jointly on the Frisian tablet, three of which were filmed ${ }^{2}$. During those sessions, the detective work of the papyrologists unravelled, and the videos enabled us to keep track of their progress throughout their inquiry and to analyze the type of processes that took place during the interpretation task. The result of this investigation, with a new clear reading of the date (29AD, probably 23 rd February), as well as a completely new transcription of the text ${ }^{3}$, was presented in the Netherlands, where the tablet originates, in April 2009 (Tresoar Fries Historisch en Letterkundig Centrum, 2009). A complete new edition of the text is now available (Bowman et al., 2009).

\section{Optimally capturing the text-bearing object}

Papyrologists do not always have direct access to the original text-bearing artefact, and so digital photographs are now usually made. In the case of the Frisian Tablet, the original artefact was brought to Oxford from Leeuwarden (NL) for digitization purposes, and for further interpretation, based on the digital pictures. The approach to digitizing wooden stylus tablets was developed by Brady (Molton et al., 2003; Brady et al., 2005) and draws its inspiration from the observation of papyrologists at work. When papyrologists have access to the physical object during the transcription process, they usually lay the tablet flat in their hands, lift it at eye level making sure that a strong light source illuminates the tablet from the side and gently rock the tablet applying to it a pitch-and-yaw motion. What essentially happens during this process is that the incident light from the side causes the incisions to cast moving shadows, and the rims of the incisions to be highlighted. Each incision then appears as a contrasted juxtaposition of highlight and shadow. The lower the light is with respect to the tablet, the larger the shadow areas are, the brighter the highlights are, and the stronger the contrast between the highlights and shadows becomes. In addition to this method of enhancement of the visibility of the score marks, it is the motion of the tablet as well as the properties of the visual system which allow a continuous perception of the 
incisions, and thus enable the expert to detect the shapes and positions of the characters engraved in the wood. The shadow casting and highlights serve as signal amplifiers, and the motion allows the visual system to combine the information from the varied tablet positions; all of which contribute to revealing the 3D properties of the object in virtue of the shadow-stereo principle (Brady et al., 2005; Tarte et al., 2008). Clearly, prior knowledge, manifested in particular in the form of memory and expectation, also plays an important role in the perception of characters, words, and sentences; this is elaborated further in section 5 . The Frisian tablet was photographed ${ }^{4}$ with the intention of allowing the digital reproduction of the rocking and rolling motion before it was taken back to the Netherlands. Motions of the tablet under fixed illumination conditions are equivalent to motions of the light source around the tablet in a fixed position. Accordingly, a high resolution digital camera was set at a fixed vantage point directly above the tablet, and a light source was moved around the tablet at positions on an imaginary sphere centered at the tablet. Each light position corresponds to a pair of angles, namely the azimuth and elevation angles. Digital pictures were taken for predetermined azimuth and elevation angle positions of the light, producing the material necessary for optimal capture of the text-bearing object in order to facilitate the interpretation process. Sixteen such images were taken for each face of the Firsian tablet, front and back.

To visualize the object, a Polynomial Texture Map (PTM) (Malzbender et al., 2001) of each view of the object can be constructed; one was generated for the front of the Frisian tablet and one for the back. By adopting such a polynomial model of interpolation, it is possible not only to summarize the information contained in the collection of captured images, but also to simulate illuminations of the tablet at intermediary positions of the light, where no actual picture was taken. A freeware is readily available from the internet to visualize PTMs (Research at HP labs, 2001); it enables display of the pictured object and interactive motion of a virtual light source around it, thus digitally reproducing the real-world pitch-and-yaw motion of the tablet.

\section{Detecting the text}

\subsection{Minimizing background interferences}

In their everyday practice, papyrologists make much use of image manipulation software such as Photoshop ${ }^{5}$, as much for its layering functionality as for the image processing capabilities it offers. The individual images usually need to be enhanced in order to minimize the interference of signals that are distracting to the eye and irrelevant with regard to the emerging text. Uneven illumination and the grooves of the wood grain are examples of such interferences. They can be remedied by image processing techniques tailored to the specific context. We have implemented several such algorithms, enabling us to correct illumination and remove the grooves corresponding to the wood grain (Molton et al., 2003; Pan et al., 2004; Tarte et al., 2008). Illumination correction is achieved via homeomorphic filtering, which rebalances the brightness throughout the image. Wood grain removal is attained by Lambertian reflectance compensation. The principle of this algorithm relies upon the prop- 
erties of the wood grain. When the lighting direction is aligned with the grooves of the wood grain, the grooves do not cast shadows. In such circumstances (e.g., for the images taken with the light source located at azimuth angles $0^{\circ}$ and $180^{\circ}$ ), the variations in brightness in an image at the locations of the grooves are solely caused by the fact that the incident light reflects from the wooden surface according to the orientation of the normal to the surface, which is given by the slope of the grooves. The Lambertian reflectance compensation isolates this effect and levels the grooves up by resetting the normal to the surface as if no wood grain were present. The result is a flattening of the slopes of the grooves; in the processed images, the wood grain then appears to have been removed. Such processed images have been generated for the Frisian tablet, and the papyrologists found them helpful (c.f. fig. 1 ). These algorithms are now available through the web-based Virtual Research Environment

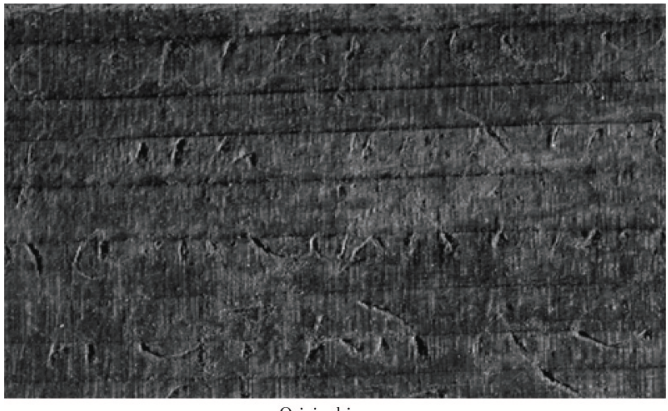

Original image

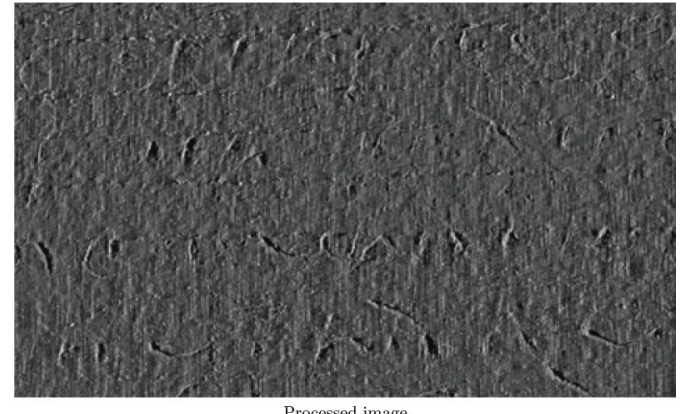

Processed image

Figure 1: Close up of the Frisian tablet. The processed image underwent illumination correction and wood grain removal.

for the Study of Documents and Manuscripts (Bowman et al., 2010) via a web-service, which takes advantage of the computational power of the National Grid Service ${ }^{6}$.

\subsection{Extracting strokelets}

Once these interferences have been removed on each of the individual images, the search for strokelets that form parts of (what may be broken) character strokes can begin. These strokelets constitute features of the image on which the human visual system locks. It has been shown, that the areas that correspond to features have specific mathematical properties. More precisely, a given function with integrability properties can be expressed as a sum of sine and cosine functions, each of which is of the form $a \cdot \sin (k \cdot t+\phi)$. The function can thus be expressed in this fashion, in what is called the Fourier (or frequency) domain. It so happens that if, for every such component of the sum $(a \cdot \sin (k \cdot t+\phi))$ the value of phase $\phi$ is the same ('congruent'), then a feature is visually detected (Morrone and Owens, 1987). Feature detection is thus achieved through the search for areas where the phase value $\phi$ is congruent. The strategy to compute such phase values $\phi$ was originally developed for 1D signals. It has recently been extended to higher dimensions (Felsberg and Sommer, 2001), and can be used to detect features in 2D signals such as images as well as in $3 \mathrm{D}$ images. 
This method, using the so-called monogenic signal to compute phase congruency, rather than an iterative search for 1D features along sampled directions (Brady et al., 2005; Pan et al., 2004; Molton et al., 2003), has had remarkable success with medical images and was implemented for the images of our tablet. Interestingly, although the images are of very high resolution and quality, the nature of the object itself has the consequence that the images were much more difficult to process than medical images, and there has been limited success in extracting the features to date. Phase congruency, based on the monogenic signal, as a feature detection method is suitable to medical images, but the type of information contained in medical images and in images of ancient documents is of a very different nature. In particular, the noise contained in the ancient document images is composed not only of the normal noise caused by the imaging technology (which can be dealt with) but also, and predominantly, of the noise due to the texture of the wood itself. This additional noise makes the task of adjusting the phase congruency's monogenic signal computational parameters extremely complex and made its performance and reliability as a method for feature detection, in our context, to be unsatisfactory so far. We are currently exploring other strategies to detect the features that a papyrologist's eyes detect with relative ease. Assuming better success with the feature detection approach in the near future, a further step was taken to help detect the text.

\subsection{Facilitating character emergence}

Here again, the aim is to base our digitized approach on evidence of practice from the papyrologists' daily work. Leaving aside for the moment the questions of expertise and prior knowledge, cognitive psychology can inform us as to how visible strokelets (i.e., detected features) are combined to form a stroke, and how strokes are combined to form a character. The completion of gaps in strokes can be seen as a problem similar to that of illusory and subjective contours, such as those demonstrated by the Kanizsa triangles (Kanizsa, 1955). There are still to date contradictory research results and debates on which criteria are used by the visual system to complete shapes: one school holds that luminance and contrast are the key components to illusory contours (Anderson et al., 2002); another school claims that tangents and orientations are the predominant criteria in the vision of illusory contours (Kellman and Shipley, 1991; Brodeur et al., 2008). We have adopted the latter, more geometrical, approach (Ullman, 1976; Rutkowski, 1979). Using the strokelets as local constraints, we have implemented a stroke completion algorithm. Three types of connections needed to be considered: smooth stroke-end to stroke-end junction, smooth stroke-end to middle-of-stroke junction, non-smooth (angular) junction. The overall strategy relies upon estimating the average orientation and proximity of strokelets. Each end of a strokelet determines a search area for potential connections. If there is a part of another strokelet within the search area, then an interpolating curve is produced to smoothly match the directions of each strokelet. If two or more search areas overlap, this suggests that there may be an angular junction. This junction is produced by creating a straight line from the centre of the overlapping area to the end of the corresponding strokelet (c.f. fig. 2). It is interesting to note here how in the edition of the Frisian tablet, the drawing tracing the 

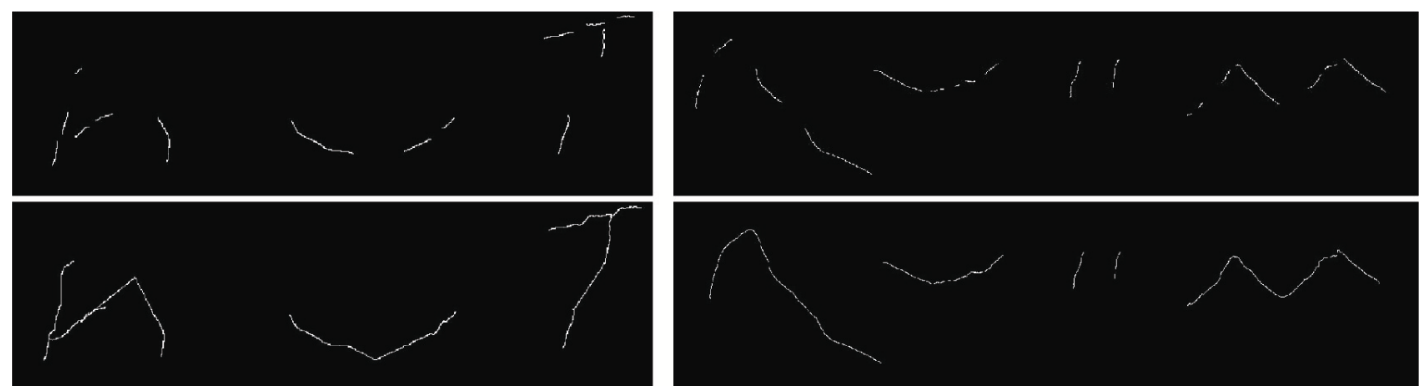

Figure 2: Examples of stroke completion, in the words 'AUT' and 'QUEM' [L4]. The input strokelets (top row) were defined manually, by tracing over the obviously visible strokelets in РНОTOSHOP(), and by further isolating (segmenting) the traced letters from the photograph.

characters as they have been interpreted does not in general distinguish between the two natures of visibility as decomposed here, namely the strokelets that are pure detectable features and the subjective contours linking the strokelets. This is one clue as to how much vision, expertise and interpretation are intertwined (c.f. section 5).

The subsequent step, once character strokes are defined, is to attempt character identification. Here, prior knowledge and strokes as features start to link up. The system will eventually propose character readings, without ever imposing them or constraining the user to pick from the digitally identified list of possible readings. Currently however, this is in a preliminary state of implementation. The encoding of characters is based on descriptors of letter shapes. Previous work that identified the type of information and vocabulary used when discussing letter forms (Terras, 2006), provided us with a framework in which an ontology of character shape can be defined. Each character can be described as a combination of strokes; each stroke has a qualitative length (short, long), a qualitative orientation (horizontal, vertical, forward and backward diagonal), and a relative position (left-of, right-of, above, below); a junction has a type (end-end, end-middle, etc...) and a qualitative angle (right, acute, obtuse). Curved lines also occur in wooden tablets, and when they do, they usually can be deconstructed into a combination of straight lines. The type of information that was not kept in this ontology is information pertaining to the ductus of the strokes; but these could be added in later. The first alphabet of letter shapes to be described in this ontology is the one compiled from the Vindolanda tablets (Bowman and Thomas, 1983, 1994, 2003); it is currently being processed and will serve as a knowledge base.

In many ways, the work on perception presented so far is akin to forensic science work, gathering clues and using advanced technologies to make them more eloquent, when the papyrologists are the actual investigators. In the next section, we introduce our initial steps in trying to understand the thought processes involved in the interpretation work, that is, the detective work itself. 


\section{Building meaning}

A model of the detective work that papyrologists undertake when confronted with the task of interpreting a document was proposed by Terras (Terras, 2005). This model identifies several levels of reading, starting at the feature (or strokelet) level, going then to the character level, the sequence of characters level, the morphemic or word level, the grammatical level, the meaning of a word level, the meaning of a group of words level, and ending at the meaning of the whole document level. Each so-defined level of reading adds meaning to the level below. Knowledge elicitation techniques also enabled Terras to determine that interpretation as a meaning-building process does not start at the feature level, progressively adding meaning while going up the levels of reading; rather it jumps from one level of reading to another, each piece of interpretation at a given level having the potential to inform another piece of interpretation at another level. This recursive process constitutes the core of the interpretation effort. What interests us particularly here, is to try and understand how and why the jumps between reading levels occur, and to what extent vision, expertise and interpretation are intertwined. In order to illustrate our discourse about some of the identified underlying mechanisms or strategies of interpretation, we recount here how a puzzling letter form in the Frisian tablet came to be read. The portions of transcript in section 5.1 deal with building an interpretation for this letter form; they are extracted from the video of the first joint interpretation session.

\subsection{A puzzling letter form}

In the following transcript, each papyrologist is identified by $(\mathrm{P} n)$; [ $L n]$ refers to a line on the front of the Frisian tablet; quoted capitalized letters correspond to Latin readings of characters, words or groups of words; and centered '...' denote eluded dialogue.

(P1): [L4] Something 'D QUEM, ${ }^{7}$.

(P2): [L1] 'GARGILIUS SECUNDI'. The last letter looks like an 'I' or an 'S', but not like a ' $U$, 8 .

(P1) flips through his stack of printed photographs in search for one where the character in question is the most visible; finds one and hands it to (P2) pointing at the letter.

(P2): It's an 'E'. After the ' $D$ ' in 'SECUNDUS' there is a zig-zag which looks like an ' $E$ '.

10 (P1): Not the two ticks kind of ' $\mathrm{E}$ ' then. It looks like a sort of ' $H$ ' or ' $\mathrm{'}$ '. We don't want an ' $H$ ' after a ' $D$ ' . Similarly, [L4] if you agree with 'QUEM' you don't want a ' $D$ ' before it... unless it's 'AD QUEM'? It could be...

(P2): It's the kind of thing you would find in a legal text, really.

(P2): We really need to establish some words to give us the letter forms. 
(P2): [L10] The first letter is bound to be a ' $Q$ ', .

(P1) disagrees with the identification of some letter shapes as ' $E$ 's as they were identified in the 1917 edition.

(P2): 'RD'? 'DIT'? 'DTI'? 'RTI'? then 'TUS' and 'VIT". Yes 'TUS'.

(P1) comments on the image quality; is happy about it.

(P2): 'VIT' has a verbal ending ring to it.

(P1): I'm note sure about those ' $R$ ' $s$, they're very square.

(P2): It could be ' $R$ ' or ' $T I$ '.

(P1): You see 'IUS' at the end and then... 'LIUS'? 'GIUS'?

(P2) pointing at the letters while spelling them out: ' $Q$ ' ' $U$ ' ' $E$ ' ' $D$ ' ' $R$ ' ' $E$ ' ' $T$, ' $U$ ' ' $\mathrm{S}$ ', 'QUEDRETUS'.

(P3) points at the problem letter on the screen.

(P2) wonderingly: 'QUADRATUS'? Read them as 'A's instead of 'E's?

30 Laughs.

(P2): 'QUADRATUS VIT, 10 .

(P1): I'm not convinced. Is this a stroke or damage? 'TUS'? 'IUS'?

All agree on the 'US' part.

(P2) pointing at the problem character, the one after ' $Q U$ ': It's definitely a vowel. It ought to be an ' $E$ '. If it were a fourth century text...

(P2) excitedly, pointing at the beginning of the line: [L6] What if we read that as 'ACTUM'? The 'CTUM' is fine!

(P1): I see what you mean!

(P2): A legal document ought to have an 'ACTUM' followed by a place name. Pause.

(P1) puzzled: Yes, it's a pity about those things that look like they might want to be 'A's.

Longer pause; the following words when spoken are in a softer quieter voice.

(P1) seems at a loss, looks at the back of the tablet to see what the letter shapes look like in spite of them having been traced by different hands from the one that wrote the text on the front of the tablet... Where do you find 'A's that look like that? You can see how you might make an 'A' like that, but...

(P3) shows a similar letter shape on the back of the tablet ${ }^{11}$.

(P1): [L10] I rather like 'QUADRATUS' although I'm still in doubt about those

'A's.

(P2): [L4] 'AD QUEM, 12 .

(P1): Yes!

(P2): If that is an ' $E$ ' (points at ' $E$ ' in 'QUEM') it doesn't make that (points at the problem letter in what might be ' $A D$ ') into a good ' $\mathrm{E}$ '.

(P3): On the back, this 'CAIUS'13 has the same form.

(P1): Yes, yes! Excellent!

(P2) describes in words and draws at the same time, showing how an 'A' could be drawn as the puzzling letter. It's a change that occurs much later but...

(P1): It's only a late looking ' $A$ ' because we think it's a late looking 'A'. We keep coming across this, things turning out to occur earlier than we thought.

(P2) draws the problem character again, and traces over it several times: This strange 


\footnotetext{
of possible senses in [L10] 'QUADRATUS' and in [back-L1-left] 'CASSIUS'14. (P1): Yes, there's got to be 'A's in this text. In fact you can see some very similar shapes, like there [L1]. Gets up to trace the letter on the screen with his finger, traces over the second character of the line ${ }^{15}$ and goes back to sit. You can almost read it; you can certainly make it out; that form looks plausible to me. If it's not that, then where are the 'A's? That's persuasive. There's got to be 'A's in this text; it's bound to be... I think that's right, I think that must be an 'A' ... So it's a fourth century 'A' in a first century text!!?!! (c.f. fig. 3)
}

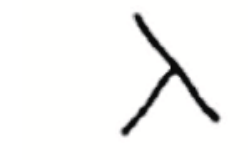

1st century $\mathrm{AD}$ 'A'

(Old Roman Cursive)

\section{n}

Frisian tablet 'A'

\section{u}

4 th century $\mathrm{AD}$ ' $\mathrm{A}$ '

(New Roman Cursive)

Figure 3: Shapes of 'A' elucidating (P2)'S "If it were a fourth century text..." and (P1)'S "So it's a fourth century 'A' in a first century text!!?!!". 1st century AD and 4th century AD 'A's from (Bowman and Thomas, 1983).

These excerpts from the video transcript retrace the story of the ' $A$ ' as it unravelled ${ }^{16}$. At the stage where the last excerpt ends, the papyrologists have almost accepted the puzzling letter form as an ' $A$ '. A little later, the reading of the end of the first line emerged as 'SECUNDAE' instead of 'SECUNDUS N', where the problematic letter after the 'D' turned out to be another occurrence of the puzzling 'A' rather than the proposed 'l', 'S', 'E', 'H' or 'L' (as reported here in the second excerpt). In subsequent sessions, the papyrologists still came back to the interpretation of that letter form as an ' $A$ ' but mostly in confirming terms, rather than in doubting terms, becoming gradually more and more certain of their interpretation, until it was completely adopted, as is reflected by the new edition of the text (Bowman et al., 2009).

\subsection{Approaches to interpretation}

As mentioned by Terras (Terras, 2006), it is apparent that each papyrologist has developed his/her own approach to the interpretation task. What we want to stress here is how much those approaches are connected to personal skills. Each of the two strategies that have been identified so far and that are exposed below are present in (P1), (P2), and (P3). Yet each of them uses preeminently the one that takes the best advantage of what they excel at.

(P2) has a marked kinaesthetic approach to the script. On many occasions throughout the sessions he has drawn the characters or traced over them with his finger, as illustrated by the transcript of the story of the ' $A$ '- -lines 59 and 64 ). He has also extensively used LCD 
tablet and pen technology ${ }^{17}$ to trace the letters as he was seeing and interpreting them. This technology, along with the use of the digital photographs and of layers in Photoshop, allows him to transfer to the digital world what used to be his drawing of the script on a transparency overlaid on a photograph of the tablet, or his still-life type drawing of the actual object when accessible. His digital drawings of the Frisian tablet's text appear in the 2009 edition. This practice of (P2)'s relates closely to his own personal skills as an artist. His rapport with the text is one of appropriation of the text through an embodiment of the script, reconstructing the movements that the scribe made when s/he wrote the text. His descriptions of the characters usually include a description not only of the relative positions of the strokes but also their starting point and ending point, and how much force was applied to the stylus throughout, reflecting both his own knowledge as a practiced artist and his palaeographical expertise. The act of drawing serves him both as a deciphering tool and as a feedback system that allows him to check how well his mental image of the script matches the actual shape of the text.

(P1) has a predominantly philological approach to the script. His rapport with the text is similar to that taken when facing a puzzle solving task ${ }^{18}$. This is well illustrated in the story of the ' $A$ ' by lines 25 and 32 . Exactly as cruciverbalists do, he relies upon the characters that he reads as almost-certain letters and tries out different hypotheses. In contrast with (P2) who draws the letters as he sees them, i.e., with the shapes of the original script, (P1) only writes the letters in modern script on a separate piece of paper. The Leiden conventions (van Groningen, 1932) allow him to express uncertainty as to a letter reading when necessary, as if marking that a certain blank box in a crossword grid might-and only might-be filled with a given letter. In some way, (P1) seems to jump straight into the mental interpretation of the score marks as symbols that are part of a meaningful text. An intuitive knowledge of the statistical occurrence of letters in Latin, which combines puzzle solving and linguistics skills, helps him to convince others and himself that the strange letter must be an ' $A$ ' after all (lines 67 and onwards).

(P3)'s preferred approach seems to be a kinaesthetic one like (P2)'s, and although it is not clearly apparent in the excerpts reproduced here, he has confirmed this on other occasions and in discussions.

Of course those two palaeographical/kinaesthetic and philological/cruciverbalistic approaches are not mutually exclusive. (P1) also resorts to tracing letters (e.g., line 68) and (P2) also uses the philological register (e.g., line 34) to build a case for the ' $A$ '. Having identified these strategies is important in terms of the design of our Interpretation Support System. The software will need to accommodate both approaches, thereby avoiding to constrain an expert to abandon his/her preferred method. Both these strategies however, make abundant use of expertise, are driven by expectations and oscillations and are subject to aspect-shifting.

\subsection{Expertise, expectations, aspect-shifting and oscillations}

Investigative methodologies adopted to decipher an ancient or damaged text may differ, as exposed in section 5.2, yet expertise in the form of skilled vision uniformly plays an important role. Our three papyrologists have been practising text deciphering for over 20 years. As 
demonstrated in cognitive psychology (Gibson, 1953), being exposed to a specialised visual task trains the capacity to see. This also pinpoints the fact that there are ways-of-looking and ways-of-seeing; both are components of expertise, and experience is part of the expertisebuilding process. The experience of deciphering a given text relates to a meaning or a difficulty, and it is thereby associated with a memory and a mental representation of the situation. This memory, as part of a mental organisational map, can be triggered by another text deciphering situation that bears similarities to the first one (Wiseman and Neisser, 1974), thus drawing on prior knowledge in a large sense when building an interpretation. Prior knowledge is composed not only of scholarly content but also of visual skill. This kind of knowledge is akin to what art historians call Morellian connoisseurship (Neer, 2005) and can to some extent be likened to pattern matching; this pattern matching is the same kind of pattern matching as the one we try to reproduce when we define a letter shape ontology (c.f. section 4.3), and that is extensively used when identifying two occurrences of a similar shape as the same character. In parallel with, and as an effect of expertise building, expectations in a phenomenological sense emerge (Ebitz, 1988; Cohn, 2007). The story of the ' $A$ ' gives a good example of expectations as they arise during the interpretation effort. The consensus about the tablet when the session starts is that it probably is a first century tablet. As a consequence, and in virtue of their palaeographical knowledge, all three papyrologists expect 'A's to have a certain shape (c.f. fig. 3). When the possibility that the 'A's in this text might resemble fourth century 'A's arises, there is a clash of expectations that expresses itself as a time conflict that needs resolving; which (P1) attempts to do when he says: "It's only a late looking 'A' because we think it's a late looking 'A'." (line 61). Throughout the interpretation process, such expectations continually arise and vanish (exactly like expectations arise and vanish when reading a literary text (Heap, 1977), although in our case the reading is far from linear). In our example, the transformation of the expectation about the shape of 'A's is related to aspect-shifting. Aspect-shifting, or aspect-dawning ('Aufleuchten eines Aspekts'), is a concept that was coined by Wittgenstein (Wittgenstein, 1953) and illustrated by what he called the 'Ente-Kaninchen' (duck-rabbit). The duck-rabbit is a drawing which can be seen as representing either a rabbit or a duck, depending on how you look at the drawing. Again this sends us back to the duality between ways-of-looking and waysof-seeing (or seeing that and seeing as) (Neer, 2005). In the story of the ' $\mathrm{A}$ ', the strange letter goes from the status: "It's an 'E'." (line 8), through to: a "thing that might want to be (...)" (line 41), as if it had a life of its own, before finally reaching the state: "That must be an ' $A$ '. ..." (line 72). The transition from ' $E$ ' to ' $A$ ' is not smooth, however. The papyrologists constantly oscillate between various hypotheses, coming back to the possibility of having an ' $E$ ' (line 26) after having considered it could be an 'A' (line 12), and then again, after wondering if it's an 'A' (line 29), considering the possibility that it could be an 'E' (line 55). Such oscillations can take place within a level of reading, e.g., on the nature of a letter, on the nature of the document, or between levels of reading (which we have so far called jumps), e.g., between the nature of a letter and the date of the document, between the nature of a letter and the type of the document. Interestingly, these oscillations do not occur only between hypotheses at and between reading levels, they also happen in a much more physical sense. To-and-fro motions take place on the tablet, between the various locations of the tablet that 
are under scrutiny, jumping from $L 4$ to $L 1$, then back to $L 4$, to $L 10$, to $L 6$, then to the Back of the tablet, then back to $L 10$, then back to $L 6$, etc... Oscillations even take place at the visual system level, where it has been shown that saccades in eye movements participate in the act of reading (Rayner, 1998). These saccade movements are accountable (at least partly) for what has been called the word superiority effect (Rumelhart and McClelland, 1986). This word superiority effect is very apparent in the story of the ' $A$ ' when (P2) proposes to read 'QUADRATUS' instead of 'QUEDRETUS' (lines 27 and 29). It has the property of linking up the micro levels (feature, letter) and the macro levels (word, document), and it takes its place into a more general cognitive theory: connectionism. The word superiority effect model actually is the basis of Terras's model of papyrological reading and transcription (Terras, 2005), which we are attempting to refine by gaining an understanding of the thought processes that eventually yield interpretations of ancient texts.

We have here identified some of the mechanisms that trigger the jumps between the levels of reading. They consist of: visual skill, developed through experience; scholarly content expectations induced by prior knowledge, e.g., philological and palaeographical knowledge; aspect-shifting expressed by the tension between ways-of-looking and ways-of-seeing; and oscillations of a physical type, such as the local to global one expressing itself as the word superiority effect. Each of these mechanisms is a specific epitome of perception and, they all contribute to the building of an interpretation.

\subsection{Building a case for the ' $A$ '}

One difficulty in building a case for an interpretation is that it is all about reconstructing a meaning for which there is no accessible ground truth. The objective towards which we are tending is to facilitate the digital recording of how such a case is made. The various strands of perception identified in section 5.3 can help categorize evidence and thus set up a framework for an implementation. One further step is to look into how the percepts emanating from these perceptions are organised in order to justify commitments to pieces of interpretation. Fig. 4 is an attempt at this kind of categorization. The approach that was adopted was to mind-map the unravelling of the interpretation in order to make explicit how the case for the ' $A$ ' was built. In the diagram in fig. 4, the puzzling letter forms have been traced over in white on the relevant portions of the processed image. The portions of tablets are dispersed in the diagram in a way that reflects their positions in the full picture. Each box corresponds to a hypothesis of interpretation, the result of a perception that is already an interpretation to some level, which we call hereafter a percept (Roued Olsen et al., 2009). The percepts are tagged as resulting from one of the mechanisms described in section 5.3, namely, scholarly expectations, skilled vision and local-global oscillation in the form of the word superiority effect. One further implicit assumption that is made explicit by this graph is that some kind of pattern matching is concurrently occurring, and that progressively the highlighted puzzling letters are identified as instances of a same character. Some additional pieces of information are added in this graph. They are: contradictions, mutual support, and weak consistency. The contradictions in the diagram have all been voiced, either as doubts on the reading of a given letter, or as implicit incompatibilities between two percepts. 


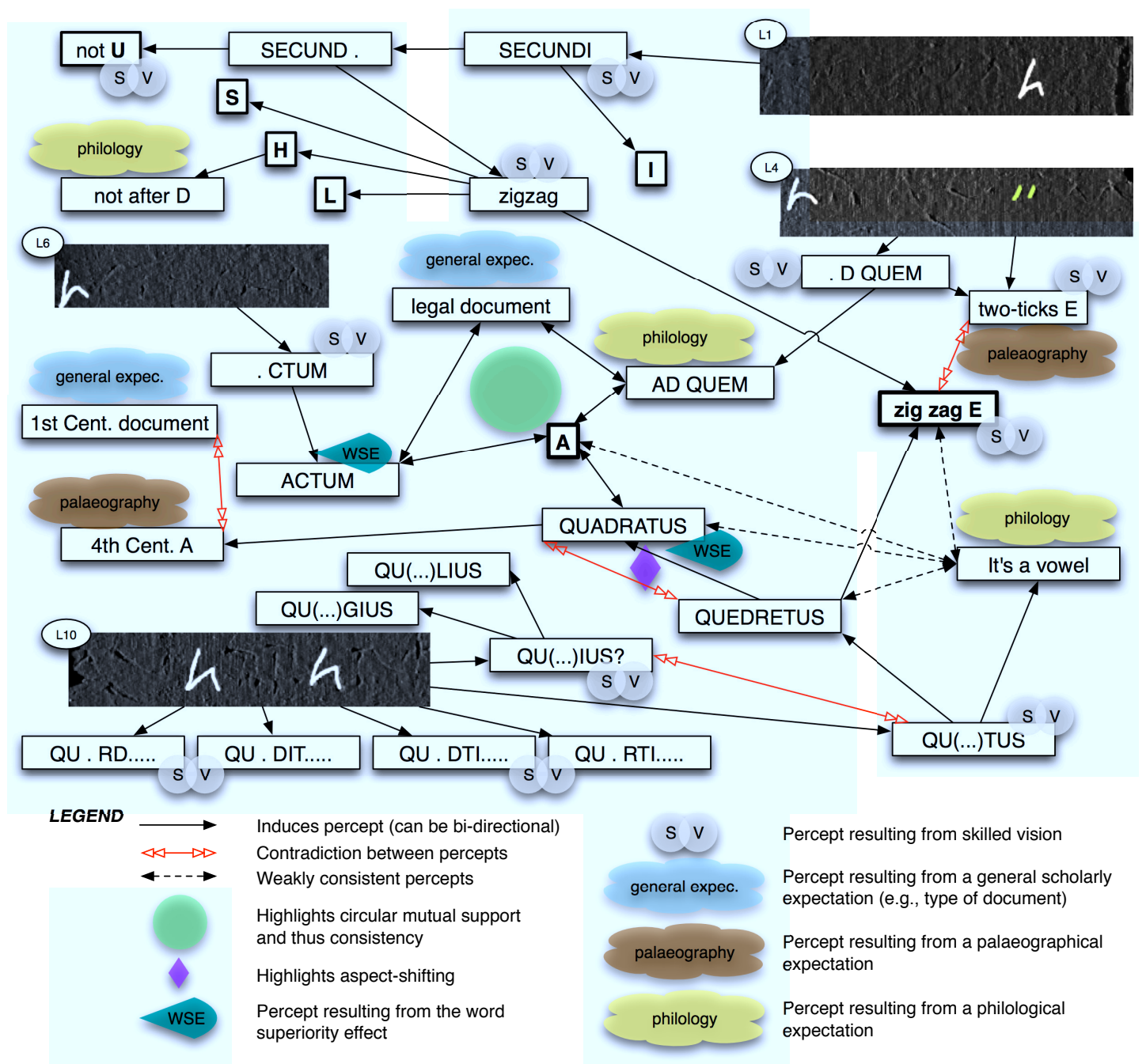

Figure 4: Schematic representation of the story of the 'A'. The premise of this diagram is the following question: 'What character is the puzzling letter form highlighted in white in the portions of photographs?'. Each box corresponds to a percept, and the thicker framed boxes are possible answers to that question.

Note the complexity of this graph, despite the fact that many elements from the transcript have been omitted. It illustrates well, on one hand how much perception and interpretation are intertwined, and on the other hand how intricate the thought process involved in the transcription task is. 
Papyrological Investigations

Mutual support is highlighted as a phenomenon where three percepts are consistent with one another, and where the circularity with respect to the identification of the puzzling letter is bi-directional. Such occurrences tend to reinforce the well-foundedness of a percept. The fact that mutual support occurs around the hypothesis ' $A$ ' and not around the hypothesis ' $E$ ' in the diagram certainly participated in eliciting the ' $A$ ' as the correct reading. Percepts that are weakly consistent with one another are also indicated. Weak consistency is a type of consistency that lacks persuasive power. And here again, the fact that weak consistency mostly happens in connection with the reading of the letter form as an ' $E$ ' weighed in the final commitment to interpreting it as an ' $A$ '. Note that the core of the global aspect-shifting from ' $E$ ' to ' $A$ ' is signaled too. It occurs when one percept fires up a second percept that contradicts the first one; typically the shift from 'QUEDRETUS' to 'QUADRATUS' is such an aspect-shifting, that eventually results in perceiving as an ' $A$ ' what was first thought to be an ' $E$ '. Each percept serves thus as a piece of evidence towards or against an interpretation, and each piece of evidence has a trigger mechanism. The diagram visually builds a case for the 'A', making explicit some of the implicit processes and knowledge. In the story of the ' $A$ ', the combination of weak consistency towards ' $E$ ' together with two mutual support loops involving ' $A$ ' as a reading, made the ' $A$ ' win over the ' $E$ ' despite the time clash provoked by two incompatible expectations. Implementing an Interpretation Support System that allows us to generalize such fine-grain recording of a process, represents quite an exciting challenge. We are currently investigating argumentation theory and theory of justification in order to propose a generalized model that would allow us to consolidate the case for the ' $A$ ', by evaluating in a more formalized way how good an evidence each percept is.

\section{Conclusion}

Papyrologists resemble detectives; for each interpretation task they undertake, they build an implicit network of percepts which all contribute (positively or negatively) towards some resulting interpretation. In a first step, some of the real-world processes that papyrologists resort to have been digitally recreated. The tools that were used to achieve this transfer to the digital world successfully are: image capture, taking advantage of the shadow-stereo principle; image processing, to minimize background interferences; shape completion, based on cognitive psychology evidence; and ontology building, informed by real-world descriptions of letter shapes. Strokelet identification using feature detection techniques is currently one focus of our research efforts. In a second step, a way to expand the reading model developed by Terras (Terras, 2005) is proposed. Two strategies adopted by papyrologists have been exposed: the palaeographical/kinaesthetic approach, and the philological/cruciverbalistic approach. Both strategies are used by papyrologists, and they resort most often to the strategy that most draws on their own personal skills. The ISS will have to provide for both approaches. Some mechanisms that trigger percepts have also been identified, as illustrated by the story of the ' $A$ ' as it unravelled during the interpretation of the Frisian tablet. These mechanisms are: skilled vision, scholarly expectations, aspect shifting and oscillations. Making the percepts and mechanisms explicit in the story of the ' $A$ ' further illustrated how perception and interpretation are intertwined, and how intricate the building of a case 
for a given piece of interpretation can be. For, in the end, 'Eye and head still must agree that this - not that-is the better way to interpret the traces of [script], as philological knowledge of [the] appropriate [language] compels letters into words and words into acceptable patterns and meaningful phrases.'(Hanson, 2001). By informing the design of an ISS with percept-triggering mechanisms, the transposition of real-world tasks into the digital world will make our software tool a user-friendly and useful tool for anyone dealing with ancient, damaged and hard-to-read documents.

Future research directions will concentrate both on interface design for the ISS, and on ways to generalise the papyrological reading model by adding qualitative ways to make further explicit the well-foundedness of a percept.

\section{Funding}

This work was supported by the AHRC-EPSRC-JISC Arts and Humanities e-Science Initiative $^{19}$ [grant number AH/E00654X/1].

\section{Acknowledgements}

I would like to thank the AHeSSC, and particularly Dr S. Dunn, for their help in disseminating the results of this research ${ }^{20}$.

My thanks go also to the three papyrologists, Prof. A.K. Bowman, Dr C.V. Crowther and Dr R.S.O. Tomlin, for letting me observe and record them at work.

I am grateful to M. Moran, who worked on the portion of the project described in section 4.3 for his 4th year thesis as part of his engineering degree, under the supervision of Prof. Sir M. Brady and myself.

Finally, I am grateful to the eSAD project team members for their precious help and support: Prof. A.K. Bowman of the Faculty of Classics, University of Oxford, UK; Prof. Sir M. Brady of the Engineering Science Department, University of Oxford, UK; Dr M. Terras of the Department of Information Studies, University College London, UK; and H. Roued-Cunliffe of the Faculty of Classics, University of Oxford, UK.

\section{Notes}

${ }^{1}$ Project website: http://esad.classics.ox.ac.uk/ — last checked: April 1, 2011

${ }^{2}$ I am grateful to Prof. A.K. Bowman, Dr C.V. Crowther, R. Kirkham and J. Pybus for giving me access to those videos, which were originally taken in order to identify requirements for the development of a Virtual Research Environment for the Study of Ancient Documents (Bowman et al. 2010) and proved a valuable source of information in particular for sections 4 and 5 of this paper.

${ }^{3}$ The original transcription of the text interpreted the tablet as a contract of sale of an ox; in the new interpretation, no trace of an ox could be found (c.f. footnote 7). The new edition interprets the document as a loan acknowledgment.

${ }^{4}$ We are grateful to $\mathrm{Dr}$ C.V. Crowther for the digitization process, which he performed with a PhaseOne Lightphase H20 digital camera back mounted on a Hasselblad 501CM medium-format camera body with CFE 4/120 macro-planar lens. 


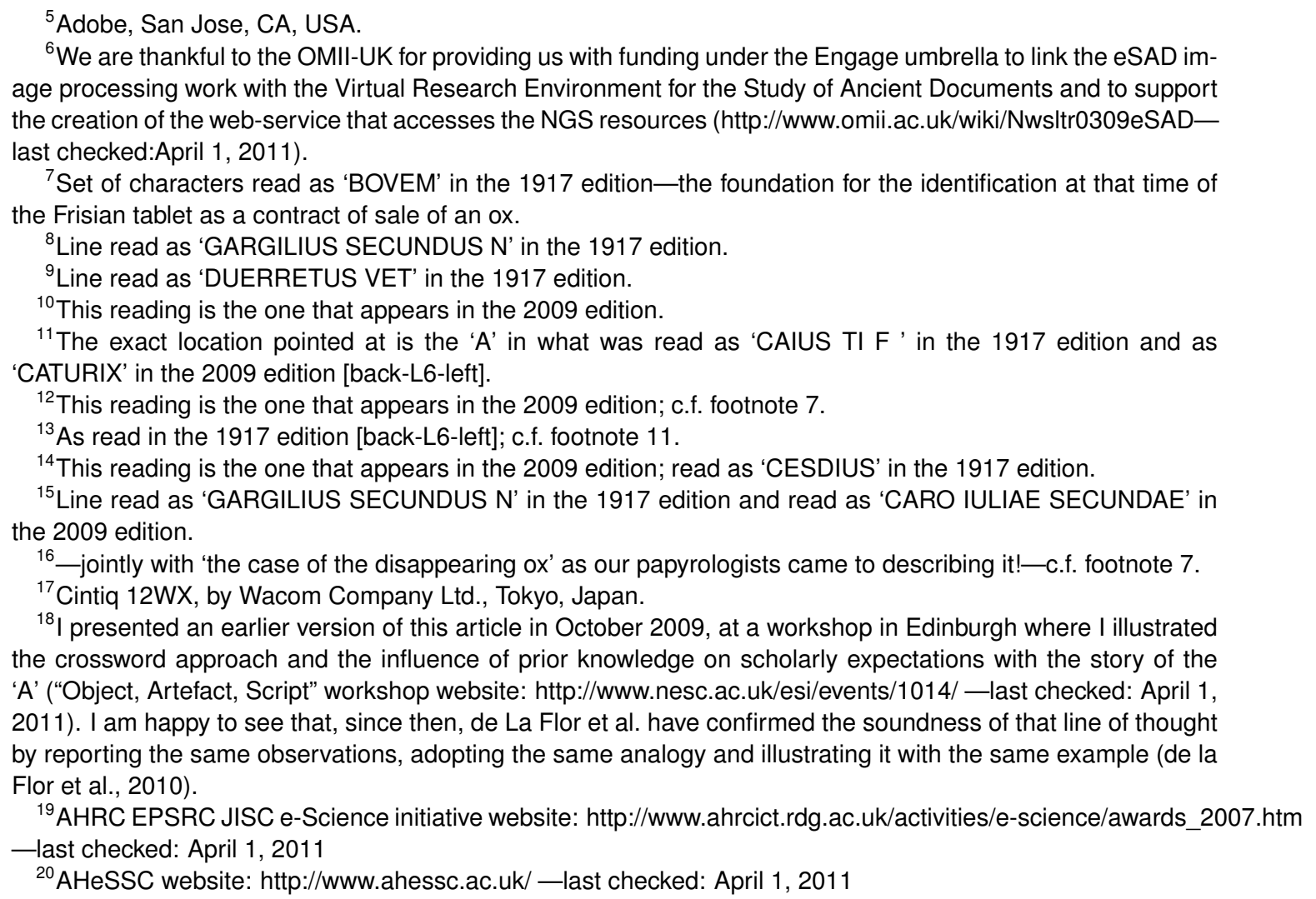

\section{References}

Anderson, B. L., Singh, M., and Fleming, R. W. (2002). The interpolation of object and surface structure. Cognitive Psychology, 44(2):148-190.

Bowman, A., Tomlin, R. S. O., and Worp, K. A. (2009). Emptio bovis Frisica: the 'Frisian ox sale' reconsidered. The Journal of Roman Studies, 99(3-4):355-66.

Bowman, A. K., Crowther, C. V., Kirkham, R., and Pybus, J. (2010). A virtual research environment for the study of documents and manuscripts. In Bodard, G. and Mahony, S., editors, Digital Research in the Study of Classical Antiquity, pages 87-104. Ashgate Press, London.

Bowman, A. K. and Thomas, J. D. (1983). Vindolanda. The Latin Writing Tablets. Britannia Monographs 4. Society for the Promotion of Roman Studies.

Bowman, A. K. and Thomas, J. D. (1994). The Vindolanda Writing Tablets (Tabulae Vindolandenses II). British Museum Press.

Bowman, A. K. and Thomas, J. D. (2003). The Vindolanda Writing Tablets (Tabulae Vindolandenses (II). British Museum Press. 
Brady, M., Pan, X., Schenck, V., Terras, M., Robertson, P., and Molton, N. (2005). Shadow stereo, image filtering and constraint propagation. In Bowman, A. K. and Brady, M., editors, Images and Artefacts of the Ancient World, volume 4 of British Academy Occasional Paper, pages 15-30. Oxford University Press/British Academy, Oxford.

Brodeur, M., Lepore, F., Lepage, M., Bacon, B. A., Jemel, B., and Debruille, J. B. (2008). Alternative mode of presentation of kanizsa figures sheds new light on the chronometry of the mechanisms underlying the perception of illusory figures. Neuropsychologia, 46(2):554-566.

Cohn, S. (2007). Seeing and drawing: the role of play in medical imaging. In Grasseni, C., editor, Skilled visions: between apprenticeship and standards, volume 6 of EASA Learning Fields, pages 91-105. Berghahn books.

de la Flor, G., Luff, P., Jirotka, M., Pybus, J., Kirkham, R., and Carusi, A. (2010). The case of the disappearing ox: Seeing through digital images to an analysis of ancient texts. In CHI '10: Proceedings of the 28th international conference on Human factors in computing systems, pages 473-482, New York, NY, USA. ACM.

Ebitz, D. (1988). Connoisseurship as practice. Artibus et Historiae, 9(18):207-212.

Felsberg, M. and Sommer, G. (2001). The monogenic signal. Signal Processing, IEEE Transactions on [see also Acoustics, Speech, and Signal Processing, IEEE Transactions on], 49(12):3136-3144.

Gibson, E. J. (1953). Improvement in perceptual judgments as a function of controlled practice or training. Psychological Bulletin, 50(6):401-431.

Hanson, A. E. (2001). Papyrology: Minding other people's business. Transactions of the American Philological Association, 131:297-313.

Heap, J. L. (1977). Toward a phenomenology of reading. Journal of Phenomenological Psychology, 8(1):103.

Kanizsa, G. (1955). Margini quasi-percettivi in campi con stimolazione omogenea. Rivista di Psicologia, 49(1):7-30.

Kellman, P. J. and Shipley, T. F. (1991). A theory of visual interpolation in object perception. Cognitive Psychology, 23(2):141-221.

Malzbender, T., Gelb, D., and Wolters, H. (2001). Polynomial texture maps. In SIGGRAPH 01: Proceedings of the 28th annual conference on Computer graphics and interactive techniques, pages 519-528, New York, NY, USA. ACM.

Molton, N., Pan, X., Brady, M., Bowman, A. K., Crowther, C., and Tomlin, R. (2003). Visual enhancement of incised text. Pattern recognition, 36(4):1031-43.

Morrone, M. and Owens, R. (1987). Feature detection from local energy. Pattern recognition letters, 6(5):303-13. 
Neer, R. (2005). Connoisseurship and the stakes of style. Critical Inquiry, 32(1):1-26.

Pan, X., Brady, M., Bowman, A., Crowther, C., and Tomlin, R. (2004). Enhancement and feature extraction for images of incised and ink texts. Image and Vision Computing, 22:443-51.

Rayner, K. (1998). Eye movements in reading and information processing: 20 years of research. Psychological Bulletin, 124(3):372-422.

Research at HP labs (2001). Polynomial texture maping. http://www.hpl.hp.com/research/ptm/-_last checked:April 1, 2011.

Roued Olsen, H., Tarte, S. M., Terras, M., Brady, J. M., and Bowman, A. K. (2009). Towards an Interpretation Support System for Reading Ancient Documents. In Digital Humanites Conference 2009, pages 237-39.

Rumelhart, D. E. and McClelland, J. L., editors (1986). Parallel Distributed Processing: Explorations in the Microstructure of Cognition, volume I. MIT Press.

Rutkowski, W. S. (1979). Shape completion. Computer graphics and image processing, 9:89-101.

Slob, E. (1998). De Koopakte Van Tolsum. The Legal History Review, 66(1-2):25-52.

Tarte, S., Brady, M., Olsen, H. R., Terras, M., and Bowman, A. (2008). Image acquisition and analysis to enhance the legibility of ancient texts. E-Science All Hands Meeting 2008.

Terras, M. (2005). Reading the readers: Modelling complex humanities processes to build cognitive systems. Lit Linguist Computing, 20:41-59.

Terras, M. (2006). Image to Interpretation. An Intelligent System to Aid Historians in Reading the Vindolanda Texts, chapter Knowledge Elicitation and the Papyrologist, pages 27119. Oxford University Press, Oxford.

Tresoar Fries Historisch en Letterkundig Centrum (2009). Schrijfplankje oudst bewaarde geschreven tekst. Tresoar: Frisian Historical and Literary Centre News:News item 31020, 24-4-2009 —last checked: April 1, 2011.

Ullman, S. (1976). Filling-in the gaps: The shape of subjective contours and a model for their generation. Biological Cybernetics, 25(1):1-6.

van Groningen, B. A. (1932). Projet d'unification des systèmes de signes critiques. Chronique d'Égypte, 7:262-269.

Vollgraff, C. W. (1917). De tabella emptionis aetatis Traiani nuper in Frisia reperta. Mnemosyne, XLV:341-52.

Wiseman, S. and Neisser, U. (1974). Perceptual organization as a determinant of visual recognition memory. The American Journal of Psychology, 87(4):675-681. 
Wittgenstein, L. (1953). Philosophische Untersuchungen, chapter II, xi.

Youtie, H. C. (1963). The papyrologist: artificer of fact. Greek, Roman and Byzantine Studies, 4(1):19-33. 